\title{
The CREATE Project: Mixed Reality for Design, Education, and Cultural Heritage with a Constructivist Approach
}

\author{
Céline Loscos ${ }^{(1)}$, Hila Ritter Widenfeld ${ }^{(1)}$, Maria Roussou ${ }^{(1,2)}$, Alexandre Meyer ${ }^{(1)}$, Franco Tecchia ${ }^{(1,6)}$, \\ George Drettakis $^{(3)}$, Emmanuel Gallo ${ }^{(3)}$, Alex Reche Martinez ${ }^{(3)}$, Nicolas Tsingos ${ }^{(3)}$, Yiorgos Chrysanthou ${ }^{(4)}$, \\ Luc Robert $^{(5)}$, Massimo Bergamasco ${ }^{(6)}$, Andrea Dettori ${ }^{(6)}$, and Souheil Soubra ${ }^{(7)}$ \\ (1) University College London, ${ }^{(2)}$ Foundation of Hellenic World, ${ }^{(3)}$ REVES/INRIA Sophia-Antipolis, \\ (4) University of Cyprus, ${ }^{(5)}$ RealViz, ${ }^{(6)}$ PERCRO, ${ }^{(7)}$ CSTB
}

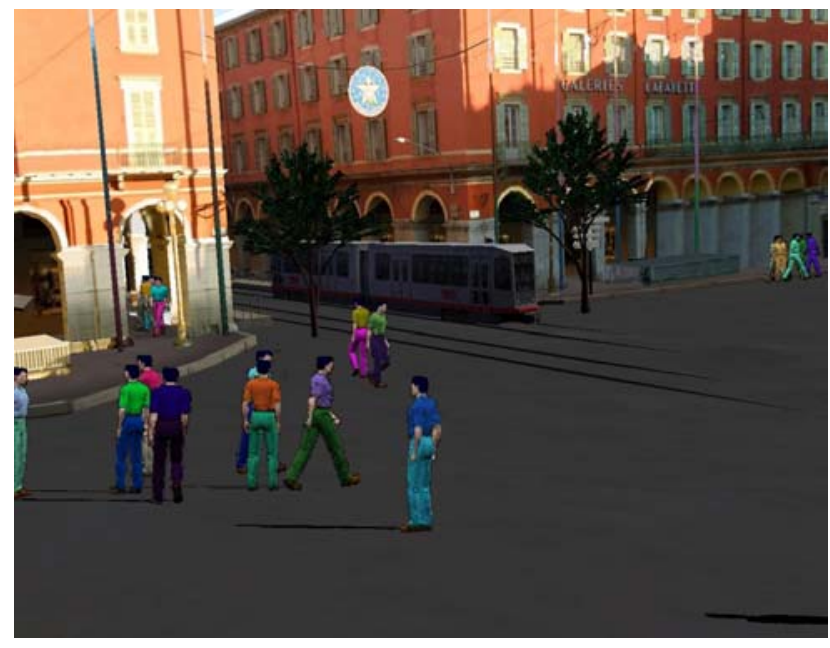

\section{Introduction}

The global scope of the CREATE project is to develop a mixed-reality framework that enables highly interactive real-time construction and manipulation of photo-realistic, virtual worlds based on real data sources. This framework will be tested and applied to cultural heritage content in an educational context, as well as to the design and review of architectural/urban planning settings. The evaluation of the project is based on a human-centered, constructivist approach to working and learning, with special attention paid to the evaluation of the resulting mixed reality experience. Through this approach, participants in an activity "construct" their own knowledge by testing ideas and concepts based on their prior knowledge and experience, applying these to a new situation, and integrating the new knowledge gained with pre-existing intellectual constructs.

Compared to previous research and design in virtual worlds, the CREATE project uses a high degree of interactivity, and includes provision for other senses (haptics and sound). The applications developed in CREATE are designed to run on different platforms, and the targeted running systems are SGI and PC driven, with immersive stereo-displays such as a workbench, a
ReaCTor ${ }^{\mathrm{TM}}$ (CAVE-like environment), and a wide projection screen.

\section{Development system and interaction tools}

In order to provide a good basis for constructive activity, the simulated environment must be authentic, meaning that it must reflect as truly as possible the familiar-to-the-user situation of the surrounding environment.

\subsection{Capture}

The first step in creating realistic mixed reality environment is the capture of real scenes. The approach we have chosen is to acquire the existing site data that will form the basis for construction and reconstruction of the virtual sites for each of the case studies. Detailed 3D reconstructions of the existing cultural or urban environment site as it is today are constructed by capturing the real scene with modeling-from-images technologies and creating appropriate 3D models. The models are then further enhanced with additional modeling to correct possible errors and complete the model.

\subsection{Interaction Tools}

We are developing a haptic interface (HI) specifically for this project that fits the tasks the user must achieve. The interface is aimed to be used by a wide range of users including children and usable in immersive stereo displays. The resulting design [1] is a resizable and robust HI's, with a large workspace and two-contact points, that enables actions, such as grabbing, rotating objects, and edge recognition, and to give the user the sense of weight, collision and roughness.

\subsection{Virtual Environment Enhancement}

To achieve a high quality immersive experience, we are developing in five main directions, that intersect to provide a coherent environment.

First, the captured data of real scenes results in a unique 3D model and a set of textures from several distinct viewpoints. To render this correctly we have implemented a prototype display application for this algorithm, which performs the correct choice of texture and the appropriate blending. 
The environment is enhanced with vegetation which is a challenge for any VR display system, since it represents a high load in polygons, and thus slows down the system. We have adapted the solution presented in [7], in which complex geometry of trees and other vegetation are replaced by points and lines when their screen projection is small with respect to the viewpoint.

An important requirement of high-quality rendering is consistent lighting between virtual, inserted elements and the captured geometry corresponding to real objects. For this we integrate the illumination from a sky and sunlight model [6], and adapt perspective shadow maps [8].

For the user interacting within a virtual city, it would be unnatural that this city is empty of life. We populate the reconstructed sites with crowds and vehicles. The population is rendered using an image-based approach [9], and lighting effects are added on vehicles [4]. We have also developed new algorithms [3][5] to represent the motion, providing more natural and smooth trajectories, consistent with the changes in the environment. The simulation is accelerated using a new occlusion culling method [2].

Finally, the inclusion of 3D spatialized sound is paramount to achieve a truly convincing mixed-reality experience. In the environments used in the CREATE project a very large number of sound sources are present. Our solution [10] is to map a large number of sound sources to a limited number of hardware channels, and is based on perceptual masking, sound source clustering and the use of graphics hardware for audio pre-mixing operations.

\section{Applications of the CREATE project}

\subsection{Understanding ancient Greek architectures}

The temple in the archaeological site of ancient Messene in Greece is being reconstructed. The site is chosen due to its cultural, educational, and symbolic significance. We identified the users of an archaelogic system to be both professional and novice users. Restoration architects and archaeologists (especially archaeology students) could use CREATE as a tool for the exploration and validation of varied reconstruction hypotheses. A highly realistic interactive environment developed through CREATE can also provide engaging experiences for students and the general public interested in learning more about history and archaeology. However, novices will most likely be attracted by the interactive learning aspects of CREATE, where the learning-by-doing and hands-on approach provides a motivating reason to visit a museum and learn in an intuitive way.

\subsection{Urban planning application}

Urban planning is a complex process involving a large number of stakeholders such as planners, developers, city administrators, community and environmental groups. Using a tool such as the one proposed through CREATE could allow stakeholders with opposing interests to explore what-if scenarios in an active and visual manner and possibly facilitate them to reach consensus on complex social, economic and environmental issues The site we have chosen is Nice in France, where an actual engineering project, the planning and development of the Tramway going through its center, is currently at the end of the public tender phase. The system will allow the presentation of several designs, allowing decision makers and local inhabitants to investigate or mix these alternatives by directly manipulating the elements concerned (pavements, traffic lights, stations, buildings, etc.).

\section{Future work}

CREATE has now been running for a year, and a first prototype is under development. In the next two years of the project, we will complete the current developed techniques and algorithms, and perform the evaluation of the system and of its learning potential.

\section{Acknowledgements}

CREATE (IST-2001-34231) is a 3-year RTD project funded by the $5^{\text {th }}$ Framework Information Society Technologies (IST) Programme of the European Union.

\section{References}

[1] A. Dettori, C.A. Avizzano, S. Marcheschi, M. Angerilli, M. Bergamasco, C.Loscos, A.Guerraz, Art Touch with the CREATE haptic interface, ICAR 2003.

[2] A. Lerner, D. Cohen-Or, Y. Chrysanthou, Breaking the Walls: Scene Partitioning and Portal Creation. Pacific Graphics 2003.

[3] C. Loscos, D. Marchal, A. Meyer. Intuitive crowd behaviour using local laws, TP.CG.' 03 .

[4] A. Meyer, C. Loscos. Multiple Environment Maps. VRST 2003.

[5] D. Michael, Y. Chrysanthou, Automatic high level avatars guidance based on affordance of movement, Eurographics 2003 (short paper session), Grenada, Spain. [6] A. J. Preetham, P. Shirley, B Smits. A Practical Analytic Model for Daylight. SIGGRAPH '99, pp 91100.

[7] M. Stamminger, G. Drettakis. Interactive Sampling and Rendering for Complex and Procedural Geometry. Eurographics Workshop on Rendering 2001.

[8] M. Stamminger, G. Drettakis, Perspective Shadow Maps, SIGGRAPH 2002.

[9] F. Tecchia, C. Loscos, Y. Chrysanthou. Visualizing crowds in real time. Computer Graphics Forum, 21(4): 753-765, Dec. 2002.

[10] N. Tsingos, E. Gallo, G. Drettakis. Perceptual Audio Rendering of Complex Virtual Environments. INRIA technical report \#4734, Feb. 2003. 